\title{
706.
}

\section{ON THE DISTRIBUTION OF ELECTRICITY ON TWO SPHERICAL SURFACES.}

[From the Philosophical Magazine, vol. v. (1878), pp. 54-60.]

IN the two memoirs "Sur la distribution de l'électricité à la surface des corps conducteurs," Mém. de l'Inst. 1811, Poisson considers the question of the distribution of electricity upon two spheres: viz. if the radii be $a, b$, and the distance of the centres be $c$ (where $c>a+b$, the spheres being exterior to each other), and the potentials within the two spheres respectively have the constant values $h$ and $g$, thenfor Poisson's $f\left(\frac{x}{a}\right)$ writing $\phi(x)$, and for his $F\left(\frac{x}{b}\right)$ writing $\Phi(x)$-the question depends on the solution of the functional equations

$$
\begin{aligned}
& a \phi(x)+\frac{b^{2}}{c-x} \Phi\left(\frac{b^{2}}{c-x}\right)=h, \\
& \frac{a^{2}}{c-x} \phi\left(\frac{a^{2}}{c-x}\right)+b \Phi(x)=g,
\end{aligned}
$$

where of course the $x$ of either equation may be replaced by a different variable.

It is proper to consider the meaning of these equations: for a point on the axis, at the distance $x$ from the centre of the first sphere, or say from the point $A$, the potential of the electricity on this spherical surface is $a \phi x$ or $\frac{a^{2}}{x} \phi\left(\frac{a^{2}}{x}\right)$, according as the point is interior or exterior; and, similarly, if $x$ now denote the distance from the centre of the second sphere (or, say, from the point $B$ ), then the potential of the electricity on this spherical surface is $b \Phi x$ or $\frac{b^{2}}{x} \Phi\left(\frac{b^{2}}{x}\right)$, according as the point is interior or exterior; $\phi(x)$ is thus the same function of $(x, a, b)$ that $\Phi(x)$ is of c. XI. 
$(x, b, a)$. Hence, first, for a point interior to the sphere $A$, if $x$ denote the distance from $A$, and therefore $c-x$ the distance of the same point from $B$, the potential of the point in question is

$$
=a \phi x+\frac{b^{2}}{c-x} \Phi\left(\frac{b^{2}}{c-x}\right) ;
$$

and, secondly, for a point interior to the sphere $B$, if $x$ denote the distance from $B$ and therefore $c-x$ the distance of the same point from $A$, the potential of the point is

$$
=\frac{a^{2}}{c-x} \phi\left(\frac{a^{2}}{c-x}\right)+b \Phi(x) .
$$

The two equations thus express that the potentials of a point interior to $A$ and of a point interior to $B$ are $=h$ and $g$ respectively.

It is to be added that the potential of an exterior point, distances from the points $A$ and $B=x$ and $c-x$ respectively, is

$$
=\frac{a^{2}}{x} \phi\left(\frac{a^{2}}{x}\right)+\frac{b^{2}}{c-x} \Phi\left(\frac{b^{2}}{c-x}\right) ;
$$

and that, by the known properties of Legendre's coefficients, when the potential upon an axial point is given, it is possible to pass at once to the expression for the potential of a point not on the axis, and also to the expression for the electrical density at a point on the two spherical surfaces respectively. The determination of the functions $\phi(x)$ and $\Phi(x)$ gives thus the complete solution of the question.

I obtain Poisson's solution by a different process as follows:-Consider the two functions

and

$$
\frac{a^{2}(c-x)}{c^{2}-b^{2}-c x},=\frac{a x+b}{c x+d}, \text { suppose, }
$$

$$
\frac{b^{2}(c-x)}{c^{2}-a^{2}-c x},=\frac{\alpha x+\beta}{\gamma x+\delta}, \text { suppose }
$$

and let the $n$th functions be

respectively.

$$
\frac{\mathrm{a}_{n} x+\mathrm{b}_{n}}{\mathrm{c}_{n} x+\mathrm{d}_{n}} \text { and } \frac{\alpha_{n} x+\beta_{n}}{\gamma_{n} x+\delta_{n}}
$$

Observing that the values of the coefficients are

$$
\left(\begin{array}{ll}
\mathrm{a}, & \mathrm{b} \\
\mathrm{c}, & \mathrm{d}
\end{array} \mid\right)\left(\begin{array}{ll}
-a^{2}, & a^{2} c \\
-c, & c^{2}-b^{2}
\end{array} \mid, \text { and }\left(\begin{array}{ll}
\alpha, & \beta \\
\gamma, & \delta
\end{array} \mid\right)\left(\begin{array}{ll}
-b^{2}, & b^{2} c \\
-c, & c^{2}-a^{2}
\end{array}\right)\right.
$$

so that we have

$$
a+d=\alpha+\delta, \quad=c^{2}-a^{2}-b^{2}, \quad a d-b c=\alpha \delta-\beta \gamma, \quad=a^{2} b^{2},
$$

and consequently that the two equations

$$
\frac{(\lambda+1)^{2}}{\lambda}=\frac{(a+d)^{2}}{a d-b c}, \quad \frac{(\lambda+1)^{2}}{\lambda}=\frac{(\alpha+\delta)^{2}}{\alpha \delta-\beta \gamma},
$$


are in fact one and the same equation

$$
\frac{(\lambda+1)^{2}}{\lambda}=\frac{\left(c^{2}-a^{2}-b^{2}\right)^{2}}{a^{2} b^{2}}
$$

for the determination of $\lambda$, then (by a theorem which $[686,687]$ I have recently obtained) we have the following equations for the coefficients

$$
\left(\begin{array}{ll}
\mathrm{a}_{n}, & \mathrm{~b}_{n} \\
\mathrm{c}_{n}, & \mathrm{~d}_{n}
\end{array} \mid, \quad\left(\begin{array}{cc}
\alpha_{n}, & \beta_{n} \\
\gamma_{n}, & \delta_{n}
\end{array} \mid\right.\right.
$$

of the $n$th functions; viz. these are:-

$$
\begin{array}{ll}
\mathrm{a}_{n} x+\mathrm{b}_{n}=\frac{1}{\lambda^{2}-1}\left(\frac{\mathrm{a}+\mathrm{d}}{\lambda+1}\right)^{n-1} & \left\{\left(\lambda^{n+1}-1\right)(\mathrm{a} x+\mathrm{b})+\left(\lambda^{n}-\lambda\right)(-\mathrm{d} x+\mathrm{b})\right\}, \\
\mathrm{c}_{n} x+\mathrm{d}_{n}=\quad, \quad \# \quad\left\{\left(\lambda^{n+1}-1\right)(\mathrm{c} x+\mathrm{d})+\left(\lambda^{n}-\lambda\right)(\mathrm{c} x-\mathrm{a})\right\}
\end{array}
$$

and similarly

$$
\begin{aligned}
& \alpha_{n} x+\beta_{n}=\frac{1}{\lambda^{2}-1}\left(\frac{\alpha+\delta}{\lambda+1}\right)^{n-1}\left\{\left(\lambda^{n+1}-1\right)(\alpha x+\beta)+\left(\lambda^{n}-\lambda\right)(-\delta x+\beta)\right\}, \\
& \gamma_{n} x+\delta_{n}=\quad, \quad \# \quad\left\{\left(\lambda^{n+1}-1\right)(\gamma x+\delta)+\left(\lambda^{n}-\lambda\right)(\gamma x-\alpha)\right\} .
\end{aligned}
$$

Observe that these equations give, as they ought to do,

$$
\mathrm{a}_{0} x+\mathrm{b}_{0}=x, \quad \mathrm{c}_{0} x+\mathrm{d}_{0}=1, \quad \mathrm{a}_{1} x+\mathrm{b}_{1}=\mathrm{a} x+\mathrm{b}, \quad \mathrm{c}_{1} x+\mathrm{d}_{1}=\mathrm{c} x+\mathrm{d} ;
$$

and similarly

$$
\alpha_{0} x+\beta_{0}=x, \quad \gamma_{0} x+\delta_{0}=1, \quad \alpha_{1} x+\beta_{1}=\alpha x+\beta, \quad \gamma_{1} x+\delta_{1}=\gamma x+\delta .
$$

Substituting in the first two equations $\frac{a^{2}}{c-x}$ in place of $x$, and in the second two equations $\frac{b^{2}}{c-x}$ in place of $x$, we obtain the following results which will be useful :-

$$
\begin{aligned}
& a_{n} a^{2}+\mathrm{b}_{n}(c-x)=a^{2}\left(\gamma_{n} x+\delta_{n}\right), \\
& \mathrm{c}_{n} a^{2}+\mathrm{d}_{n}(c-x)=\frac{1}{b^{2}}\left(\alpha_{n+1} x+\beta_{n+1}\right), \\
& a_{n} b^{2}+\beta_{n}(c-x)=b^{2}\left(\mathrm{c}_{n} x+\mathrm{d}_{n}\right), \\
& \gamma_{n} b^{2}+\delta_{n}(c-x)=\frac{1}{a^{2}}\left(a_{n+1} x+b_{n+1}\right),
\end{aligned}
$$

the last two of which are obtained from the first two by a mere interchange of letters; it will therefore be sufficient to prove the first and second equations.

For the first equation we have

$$
a_{n} a^{2}+b_{n}(c-x)=\frac{1}{\lambda^{2}-1}\left(\frac{a+d}{\lambda+1}\right)^{n-1}\left\{\left(\lambda^{n+1}-1\right)\left[a a^{2}+b(c-x)\right]+\left(\lambda^{n}-\lambda\right)\left[-d a^{2}+b(c-x)\right]\right\},
$$


where the term in \{\} is

viz. this is

$$
=\left(\lambda^{n+1}-1\right)\left[-a^{4}+a^{2} c(c-x)\right]+\left(\lambda^{n}-\lambda\right)\left[a^{2}\left(b^{2}-c^{2}\right)+a^{2} c(c-x)\right] ;
$$

or it is

$$
=a^{2}\left\{\left(\lambda^{n+1}-1\right)\left(c^{2}-a^{2}-c x\right)+\left(\lambda^{n}-\lambda\right)\left(b^{2}-c x\right)\right\} ;
$$

$$
=a^{2}\left\{\left(\lambda^{n+1}-1\right)(\gamma x+\delta)+\left(\lambda^{n}-\lambda\right)(\gamma x-\alpha)\right\},
$$

whence the relation in question.

The proof of the second equation is a little more complicated. We have

$$
\mathrm{c}_{n} a^{2}+\mathrm{d}_{n}(c-x)=\frac{1}{\lambda^{2}-1}\left(\frac{\mathrm{a}+\mathrm{d}}{\lambda+1}\right)^{n-1}\left\{\left(\lambda^{n+1}-1\right)\left[\mathrm{c} a^{2}+\mathrm{d}(c-x)\right]+\left(\lambda^{n}-\lambda\right)\left[\mathrm{c} a^{2}-\mathrm{a}(c-x)\right]\right\},
$$

where the term in \{\} is

$$
=\left(\lambda^{n+1}-1\right)\left[-c a^{2}+\left(c^{2}-b^{2}\right)(c-x)\right]+\left(\lambda^{n}-\lambda\right)\left[-c a^{2}+a^{2}(c-x)\right] .
$$

Comparing this with

$$
\alpha_{n+1} x+\beta_{n+1}=\frac{1}{\lambda^{2}+1}\left(\frac{\alpha+\delta}{\lambda+1}\right)^{n}\left\{\left(\lambda^{n+2}-1\right)(\alpha x+\beta)+\left(\lambda^{n+1}-\lambda\right)(-\delta x+\beta)\right\},
$$

where the term in \{\} is

$$
=\left(\lambda^{n+2}-1\right)\left[b^{2}(c-x)\right]+\left(\lambda^{n+1}-\lambda\right)\left[-c\left(c^{2}-a^{2}-b^{2}\right)+\left(c^{2}-a^{2}\right)(c-x)\right],
$$

it is to be observed that the quotient of the two terms in \{\} is in fact a constant; this is most easily verified as follows. Dividing the first of them by the second, we have a quotient which when $x=c$ is

$$
\frac{\left(\lambda^{n+1}-1\right)\left(-c a^{2}\right)+\left(\lambda^{n}-\lambda\right)\left(-c a^{2}\right)}{\left(\lambda^{n+1}-\lambda\right)\left\{-c\left(c^{2}-a^{2}-b^{2}\right)\right\}},=\frac{a^{2}\left(\lambda^{n+1}-1+\lambda^{n}-\lambda\right)}{\left(\lambda^{n+1}-\lambda\right)\left(c^{2}-a^{2}-b^{2}\right)},=\frac{a^{2}(\lambda+1)}{\left(c^{2}-a^{2}-b^{2}\right) \lambda},
$$

and when $x=0$ is

$$
\frac{\left(\lambda^{n+1}-1\right) c\left(c^{2}-a^{2}-b^{2}\right)}{\left(\lambda^{n+2}-1\right) b^{2} c+\left(\lambda^{n+1}-\lambda\right) b^{2} c},=\frac{\left(\lambda^{n+1}-1\right)\left(c^{2}-a^{2}-b^{2}\right)}{\left(\lambda^{n+2}-1+\lambda^{n+1}-\lambda\right) b^{2}},=\frac{c^{2}-a^{2}-b^{2}}{b^{2}(\lambda+1)}:
$$

these two values are equal by virtue of the equation which defines $\lambda$; and hence the quotient of the two linear functions having equal values for $x=c$ and $x=0$, has always the same value; say it is $=\frac{c^{2}-a^{2}-b^{2}}{b^{2}(\lambda+1)}$. Hence, observing that $a+d=\alpha+\delta$, $=c^{2}-a^{2}-b^{2}$, the quotient, $\mathrm{c}_{n} a^{2}+\mathrm{d}_{n}(c-x)$ divided by $\alpha_{n+1} x+\beta_{n+1}$, is

$$
=\frac{\lambda+1}{c^{2}-a^{2}-b^{2}} \cdot \frac{c^{2}-a^{2}-b^{2}}{b^{2}(\lambda+1)}, \quad=\frac{1}{b^{2}}
$$

or we have the required equation

$$
c_{n} a^{2}+d_{n}(c-x)=\frac{1}{b^{2}}\left(\alpha_{n+1} x+\beta_{n+1}\right) .
$$


Considering now the functional equations, suppose for the moment that $g$ is $=0$; the two equations may be satisfied by assuming

$$
\begin{aligned}
& \phi(x)=h\left\{\frac{1}{\mathrm{c}_{0} x+\mathrm{d}_{0}}+\frac{\omega}{\mathrm{c}_{1} x+\mathrm{d}_{1}}+\ldots\right\} L, \\
& \Phi(x)=-h\left\{\frac{\omega}{\alpha_{1} x+\beta_{1}}+\frac{\omega^{2}}{\alpha_{2} x+\beta_{2}}+\ldots\right\} M .
\end{aligned}
$$

We in fact, from the foregoing relations, at once obtain

$$
\begin{aligned}
& \frac{a^{2}}{c-x} \phi \frac{a^{2}}{c-x}=h\left\{\frac{\omega}{\alpha_{1} x+\beta_{1}}+\frac{\omega^{2}}{\alpha_{2} x+\beta_{2}} \cdots\right\} \frac{a^{2} b^{2} L}{\omega}, \\
& \frac{b^{2}}{c-x} \Phi \frac{b^{2}}{c-x}=-h\left\{\frac{\omega}{c_{1} x+\mathrm{d}_{1}}+\frac{\omega^{2}}{c_{2} x+\mathrm{d}_{2}} \cdots\right\} M .
\end{aligned}
$$

To satisfy the first equation we must have $M=a L$; viz. this being so, the equation becomes

$$
a \phi x+\frac{b^{2}}{c-x} \Phi\left(\frac{b^{2}}{c-x}\right)=\frac{a L h}{\mathrm{c}_{0} x+\mathrm{d}_{0}}
$$

or, since $c_{0} x+\mathrm{d}_{0}=1$, the equation will be satisfied if only $a L=1$, whence also $M=1$. And the second equation will be satisfied if only $\frac{a^{2} b^{2} L}{\omega}=b M$; viz. substituting for $L, M$ their value, we find $\omega=a b$.

Supposing, in like manner, that $h=0, g$ retaining its proper value, we find a like solution for the two equations; and by simply adding the solutions thus obtained, we have a solution of the original two equations

$$
\begin{aligned}
& a \phi(x)+\frac{b^{2}}{c-x} \Phi\left(\frac{b^{2}}{c-x}\right)=h, \\
& \frac{a^{2}}{c-x} \phi\left(\frac{a^{2}}{c-x}\right)+b \Phi(x)=g ;
\end{aligned}
$$

viz. the solution is

$$
\begin{aligned}
& \phi(x)=\frac{h}{a}\left\{\frac{1}{\mathrm{c}_{0} x+\mathrm{d}_{0}}+\frac{a b}{\mathrm{c}_{1} x+\mathrm{d}_{1}}+\ldots\right\}-g\left\{\frac{a b}{\mathrm{a}_{1} x+\mathrm{b}_{1}}+\frac{(a b)^{2}}{\mathrm{a}_{2} x+\mathrm{b}_{2}}+\ldots\right\}, \\
& \Phi(x)=-h\left\{\frac{a b}{\alpha_{1} x+\beta_{1}}+\frac{(a b)^{2}}{\alpha_{2} x+\beta_{2}}+\ldots\right\}+\frac{g}{b}\left\{\frac{1}{\gamma_{0} x+\delta_{0}}+\frac{a b}{\gamma_{1} x+\delta_{1}}+\ldots\right\} .
\end{aligned}
$$

We have a general solution containing an arbitrary constant $P$ by adding to the foregoing values for $\phi x$ a term

and for $\Phi x$ a term

$$
=\frac{P b(a-b)}{\sqrt{a^{2}(c-x)-x\left(c^{2}-b^{2}-c x\right)}},
$$

$$
=\frac{P a(b-a)}{\sqrt{b^{2}(c-x)-x\left(c^{2}-a^{2}-c x\right)}},
$$


as may be easily verified if we observe that the function

$$
a^{2}(c-x)-x\left(c^{2}-b^{2}-c x\right),
$$

writing therein $\frac{a^{2}}{c-x}$ for $x$, becomes

$$
=\frac{a^{2}}{(c-x)^{2}}\left\{b^{2}(c-x)-x\left(c^{2}-a^{2}-c x\right)\right\}:
$$

and similarly that

$$
b^{2}(c-x)-x\left(c^{2}-a^{2}-c x\right)
$$

writing therein $\frac{b^{2}}{c-x}$ for $x$, becomes

$$
=\frac{b^{2}}{(c-x)^{2}}\left\{a^{2}(c-x)-x\left(c^{2}-b^{2}-c x\right)\right\} .
$$

More generally, the terms to be added are for $\phi x$ a term as above, where $P$ denotes a function of $x$ which remains unaltered when $x$ is changed into $\frac{a^{2}(c-x)}{c^{2}-b^{2}-c x}$, and for $\Phi x$ a term as above with $P^{\prime}$ instead of $P$, where $P^{\prime}$ denotes what $P$ becomes when $x$ is changed into $\frac{a^{2}}{c-x}$. But these additional terms vanish for the electrical problem, and the correct values of $\phi x, \Phi x$ are the particular values given above.

It is to be remarked that the function

$$
\frac{a^{2}(c-x)}{c^{2}-b^{2}-c x} \text { is }=\frac{a^{2}}{c-\frac{b^{2}}{c-x}}
$$

viz. considering $x$ as the distance of a point $X$ from $A$, then taking the image of $X$ in regard to the sphere $B$, and again the image of this image in regard to the sphere $A$, the function in question is the distance of this second image from $A$. And similarly the function

$$
\frac{b^{2}(c-x)}{c^{2}-a^{2}-c x} \text { is }=\frac{b^{2}}{c-\frac{a^{2}}{c-x}} ;
$$

viz. considering here $x$ as the distance of the point $X$ from $B$, then taking the image of $X$ in regard to the sphere $A$, and again the image of this image in regard to the sphere $B$, the function in question is the distance of this second image from $B$. It thus appears that Poisson's solution depends upon the successive images of $X$ in regard to the spheres $B$ and $A$ alternately, and also on the successive images of $X$ in regard to the spheres $A$ and $B$ alternately. This method of images is in fact employed in Sir W. Thomson's paper "On the Mutual Attraction or Repulsion between two Electrified Spherical Conductors," Phil. Mag., April and August, 1853. 